\title{
On microscopic theory of spin- $S$ Bose-Einstein condensate in a magnetic field
}

\author{
A.S. Peletminskii ${ }^{1,2}$, S.V. Peletminskii ${ }^{1}$, Yu.V. Slyusarenko ${ }^{1}$ \\ ${ }^{1}$ Akhiezer Institute for Theoretical Physics, National Science Center "Kharkov \\ Institute of Physics and Technology", 61108 Kharkov, Ukraine \\ ${ }^{2}$ The Abdus Salam International Centre for Theoretical Physics, 34100 Trieste, Italy \\ E-mail: spelet@kipt.kharkov.ua
}

\begin{abstract}
.
The Bogoliubov model for weakly interacting Bose gas is extended to Bose-Einstein condensation (BEC) of spin-S atoms in a magnetic field. Equation for the vectorial order parameter valid at temperature $T \rightarrow 0$ is derived and its particular solution is found. This solution corresponds to the formation of BEC of atoms with a definite spin projection onto direction of a magnetic field. We study the thermodynamic stability of the found solution and obtain the expressions for low-lying collective modes.
\end{abstract}

PACS numbers: 05.30.d; 05.30.Jp; 03.75.Fi

Keywords: Bose-Einstein condensation, Spinor condensate, Weakly interacting Bose gas, Quasiaverages, Thermodynamics, Excitation spectra 


\section{Introduction}

After the first remarkable experiments concerning the observation of BEC in dilute gases of alkali atoms such as ${ }^{87} \mathrm{Rb}[1],{ }^{23} \mathrm{Na}[2]$, and ${ }^{7} \mathrm{Li}$ [3] the interest to this phenomenon has revived [4, 5]. Later on, BEC has been also obtained in other atomic species: atomic hydrogen [6], metastable ${ }^{4} \mathrm{He}$ [7], and ${ }^{41} \mathrm{~K}$ [8]. The experimental realization of BEC has become possible due to the progress of laser cooling and trapping techniques [9]. The carried out experiments have proved many predictions of the microscopic theory for weakly interacting Bose gas, which originates from the pioneering work of Bogoliubov [10]. Bogoliubov's theory has become almost the first theory in which it was necessary to move essentially from the methods of standard perturbative approach while describing the interaction effects. However, this theory, in its original formulation, did not take into account the internal degrees of freedom of atoms. The effect of spin degrees of freedom for weakly interacting Bose gas (spinor BEC) has been studied in [11]-[19].

The realization of optical trapping for atomic condensate [20] has stimulated theoretical interest to spinor BEC. Bose condensation in a weakly interacting gas of bosonic atoms has been studied theoretically by many authors both for spin-1 [12]-[17] and spin-2 [18], 19] bosons. These investigations are based on the effective interaction Hamiltonians of two bosons, in which the interaction is characterized by a definite number of interaction constants $-s$-wave scattering lengths. The number of scattering lengths is determined by the total spin of two interacting bosons taking into account the symmetry properties of their wave function. For example, in case of spin-1 atoms the interaction Hamiltonian contains two interaction constants [12]-[17], in case of spin-2 atoms there are three interaction constants [18], [19]. Thus, as the spin value of atoms grows, the number of constants, which characterize the interaction of two bosons, is increased. Note that in the mentioned effective Hamiltonians it is difficult to interpret the physical nature of the separated term of non-relativistic interaction not associated with neither potential nor spin-exchange interactions (see e.g. [18]).

In this paper we study a weakly interacting Bose gas of particles with arbitrary integer spin $S$ in a magnetic field (see also [11]). We start from the microscopic interaction Hamiltonian for two spin- $S$ bosons. This Hamiltonian is specified by two functions, which describe potential and spin-exchange interactions of spin- $S$ atoms. According to general rules of quantum mechanics, we pass from the pairwise interaction of two bosons to the standard expression for binary interaction of arbitrary number of bosons in the second quantization representation. By solving the multichannel scattering problem for the considered Hamiltonian we could find, in principle, all scattering lengths in terms of the functions characterizing the potential and spin-exchange interactions. Thereby, it would be possible to obtain the Hamiltonians analogous to the above mentioned effective interaction Hamiltonians (see e.g. [18]). However, the use of the microscopic Hamiltonian enables to restrict ourself by two interaction constants even in the case of arbitrary spin when studying the ground state, stability, and excitations in a weakly interacting gas in the presence of BEC. 


\section{Method of quasiaverages and the model with a separated condensate}

To describe the system with a spontaneously broken symmetry we address to the method of quasiaverages [21, 22]. According to this method the Gibbs statistical operator is modified so that it possesses the symmetry of degenerate state. This modification is usually done by introducing the infinitesimal "source" $\nu \hat{F}(\nu \rightarrow 0)$ into the Gibbs exponent, which has the symmetry of phase under consideration. Then, the average value of any physical quantity $\hat{A}$ is defined as

$$
\prec \hat{A} \succ=\lim _{\nu \rightarrow 0} \lim _{V \rightarrow \infty} \operatorname{Tr} \hat{w}_{\nu} \hat{A},
$$

where the Gibbs statistical operator $\hat{w}_{\nu}$ has the form

$$
\hat{w}_{\nu}=\exp \left(\Omega_{\nu}-\beta(\hat{H}-\mu \hat{N}+\nu \hat{F})\right) .
$$

Here $\beta=1 / T, \mu$ are the reciprocal temperature and chemical potential respectively and $\hat{H}, \hat{N}$ are the system Hamiltonian and the particle number operator. The thermodynamic potential $\Omega_{\nu}$ being a function of thermodynamic parameters $\beta, \mu$ is found from the normalization condition $\operatorname{Tr} \hat{w}_{\nu}=1$. Notice that the limits in (1) are not permutable.

Consider a gas of condensed bosonic atoms with spin $S$. The formation of a condensate is accompanied by the gauge symmetry breaking and, therefore, in order to remove this kind of degeneracy we should choose the "source" $\nu \hat{F}$ in (2) such that $\left[\hat{w}_{\nu}, \hat{N}\right] \neq 0$ ( $\hat{N}$ is the generator of phase transformation),

$$
\nu \hat{F}=\nu_{\alpha} \int d^{3} x\left(\hat{\psi}_{\alpha}^{\dagger}(\mathbf{x})+\hat{\psi}_{\alpha}(\mathbf{x})\right)
$$

where $\hat{\psi}_{\alpha}^{\dagger}(\mathbf{x}), \hat{\psi}_{\alpha}(\mathbf{x})$ are the creation and annihilation operators with index $\alpha$ taking $2 S+1$ values (the summation over repeated indices is assumed). Then, according to (1), (2),$\prec \hat{\psi}_{\alpha}(\mathbf{x}) \succ=V^{-1 / 2} \prec \hat{a}_{0 \alpha} \succ \sim 1$ that corresponds to the formation of atomic condensate with momenta $\mathbf{p}=0$. The order parameter $\Psi_{\alpha}=V^{-1 / 2} \prec \hat{a}_{0 \alpha} \succ$ is called the condensate wave function.

The method of quasiaverages and the spatial correlation decay principle enable to justify the replacement of creation and annihilation operators of atoms with momentum $\mathbf{p}=0$ by $c$-numbers, $\hat{a}_{0 \alpha}, \hat{a}_{0 \alpha}^{\dagger} \rightarrow \sqrt{V} \Psi_{\alpha}, \sqrt{V} \Psi_{\alpha}^{*}$ [21]-[23] (the condensate separation procedure).

The basic statement of the method of quasiaverages applied to the description of BEC consists in the following [21]-[23]: the Gibbs statistical operator is replaced by

$$
\hat{w}(\Psi)=\exp (\Omega(\Psi)-\beta(\hat{H}(\Psi)-\mu \hat{N}(\Psi))),
$$

where $\Psi=\left\{\Psi_{\alpha}, \Psi_{\alpha}^{*}\right\}$ is found from the following equation:

$$
\frac{\partial \Omega(\Psi)}{\partial \Psi}=0
$$




\section{The ground state of spin- $S$ condensate in a magnetic field}

In this section we study one of the possible ground states of spin- $S$ BEC in a magnetic field. In doing so, we start from the Hamiltonian $\hat{\mathcal{H}}=\hat{H}-\mu \hat{N}$, which determines the Gibbs statistical operator (41) and has the following form:

$$
\hat{\mathcal{H}}=\hat{\mathcal{H}}_{0}+\hat{\mathcal{H}}_{\mathrm{p}}+\hat{\mathcal{H}}_{\mathrm{e}}
$$

where

$$
\begin{aligned}
& \hat{\mathcal{H}}_{0}=\sum_{\mathbf{p}} \hat{a}_{\mathbf{p} \alpha}^{\dagger}\left[\left(\varepsilon_{p}-\mu\right) \delta_{\alpha \beta}-\mathbf{h} \mathbf{S}_{\alpha \beta}\right] \hat{a}_{\mathbf{p} \beta}, \quad \varepsilon_{p}=\frac{p^{2}}{2 M} \\
& \hat{\mathcal{H}}_{\mathrm{p}}=\frac{1}{2 V} \sum_{\mathbf{p}_{1} \ldots \mathbf{p}_{4}} U\left(\mathbf{p}_{13}\right) \delta_{\mathbf{p}_{1}+\mathbf{p}_{2}, \mathbf{p}_{3}+\mathbf{p}_{4}} \hat{a}_{\mathbf{p}_{1} \alpha}^{\dagger} \hat{a}_{\mathbf{p}_{2} \beta}^{\dagger} \hat{a}_{\mathbf{p}_{3} \alpha} \hat{a}_{\mathbf{p}_{4} \beta}, \\
& \hat{\mathcal{H}}_{\mathrm{e}}=\frac{1}{2 V} \sum_{\mathbf{p}_{1} \ldots \mathbf{p}_{4}} J\left(\mathbf{p}_{13}\right) \delta_{\mathbf{p}_{1}+\mathbf{p}_{2}, \mathbf{p}_{3}+\mathbf{p}_{4}} \hat{a}_{\mathbf{p}_{1} \alpha}^{\dagger} \hat{a}_{\mathbf{p}_{2} \beta}^{\dagger} \mathbf{S}_{\alpha \gamma} \mathbf{S}_{\beta \rho} \hat{a}_{\mathbf{p}_{3} \gamma} \hat{a}_{\mathbf{p}_{4} \rho} .
\end{aligned}
$$

Here $\mathbf{S}_{\alpha \beta}$ are the spin matrices, $U\left(\mathbf{p}_{13}\right), J\left(\mathbf{p}_{13}\right)\left(\mathbf{p}_{13}=\mathbf{p}_{1}-\mathbf{p}_{3}\right)$ are the Fourier transforms of the amplitudes of potential and spin-exchange interactions respectively, and $\mathbf{h}=g \mathbf{H} / S$ ( $g$ is the Bohr magneton, and $\mathbf{H}$ is an external magnetic field). For our next calculations it is convenient to introduce the so-called ladder operators $\hat{S}_{ \pm}=\hat{S}_{x} \pm i \hat{S}_{y}$. Then, their nonzero matrix elements in the representation, where $\hat{S}_{z}$ is a diagonal matrix, $\left\langle\alpha\left|\hat{S}_{z}\right| \alpha^{\prime}\right\rangle=\alpha \delta_{\alpha \alpha^{\prime}}$, have the form

$$
\begin{aligned}
& \left\langle\alpha+1\left|\hat{S}_{+}\right| \alpha\right\rangle=\sqrt{S(S+1)-\alpha(\alpha+1)}, \\
& \left\langle\alpha-1\left|\hat{S}_{-}\right| \alpha\right\rangle=\sqrt{S(S+1)-\alpha(\alpha-1)} .
\end{aligned}
$$

Now we separate the $\mathbf{p}=0$ components $\hat{a}_{0 \alpha}$ in the Hamiltonian (the replacement of $\hat{a}_{0 \alpha}$ by $c$-numbers, $\hat{a}_{0 \alpha} \rightarrow \sqrt{V} \Psi_{\alpha}$ ) and keep the terms only up to second order in $\hat{a}_{\mathbf{p} \alpha}$. We omit the higher order terms, since they should be taken into account only when examining the interaction between quasiparticles, which we will introduce in the next section. As a result the Hamiltonian takes the form $\hat{\mathcal{H}} \approx \mathcal{H}^{(0)}+\hat{\mathcal{H}}^{(2)}$. The explicit expression for $\mathcal{H}^{(0)}$, which contains only $c$-numbers $\Psi_{\alpha}$ reads

$$
\frac{\mathcal{H}^{(0)}}{V}=\frac{U(0)}{2}\left(\Psi^{*} \Psi\right)^{2}+\frac{J(0)}{2}\left(\Psi^{*} \hat{\mathbf{S}} \Psi\right)^{2}-\mathbf{h} \Psi^{*} \hat{\mathbf{S}} \Psi-\mu \Psi^{*} \Psi
$$

where

$$
\Psi^{*} \Psi=\Psi_{\alpha}^{*} \Psi_{\alpha}, \quad \Psi^{*} \hat{\mathbf{S}} \Psi=\Psi_{\alpha}^{*} \mathbf{S}_{\alpha \beta} \Psi_{\beta}
$$

The explicit form for $\hat{\mathcal{H}}^{(2)}$ will be written in the next section.

Next, making use the normalization condition $\operatorname{Tr} \hat{w}=1$, we find immediately the thermodynamic potential density $\omega=\Omega T / V$ in the leading approximation (neglect of quasiparticles; $T \rightarrow 0$ ) of the model for weakly interacting Bose gas,

$$
\omega=\frac{U(0)}{2}\left(\Psi^{*} \Psi\right)^{2}+\frac{J(0)}{2}\left(\Psi^{*} \hat{\mathbf{S}} \Psi\right)^{2}-\mathbf{h} \Psi^{*} \hat{\mathbf{S}} \Psi-\mu \Psi^{*} \Psi .
$$

Therefore, Eq. (5) for $\Psi_{\alpha}$ takes the form

$$
\mu \Psi_{\alpha}-U(0)\left(\Psi^{*} \Psi\right) \Psi_{\alpha}-J(0)\left(\Psi^{*} \hat{\mathbf{S}} \Psi\right) \mathbf{S}_{\alpha \beta} \Psi_{\beta}+\mathbf{h} \mathbf{S}_{\alpha \beta} \Psi_{\beta}=0 .
$$


If to introduce the normalized spin functions $\zeta_{\alpha}, \Psi_{\alpha}=\sqrt{n} \zeta_{\alpha}$, where $n=\Psi_{\alpha} \Psi_{\alpha}^{*}$ is the condensate density and $\zeta_{\alpha} \zeta_{\alpha}^{*}=1$, then the latter equation is written as

$$
\mu \zeta_{\alpha}-n U(0) \zeta_{\alpha}-n J(0)\left(\zeta^{*} \hat{\mathbf{S}} \zeta\right) \mathbf{S}_{\alpha \beta} \zeta_{\beta}+\mathbf{h} \mathbf{S}_{\alpha \beta} \zeta_{\beta}=0 .
$$

Assuming the vector $\mathbf{h}$ directed along $z$-axis $(\mathbf{h}=(0,0, h))$, its solution $\zeta_{\alpha}^{(m)}$ being an eigenfunction of $\hat{S}_{z},\left(\hat{S}_{z}\right)_{\alpha \beta} \zeta_{\beta}^{(m)}=m \zeta_{\alpha}^{(m)}$, has the form

$$
\zeta_{\alpha}^{(m)}=\delta_{\alpha m}
$$

Next, taking into account that $\hat{S}_{z}$ is a diagonal matrix, whereas $\hat{S}_{ \pm}$have no diagonal matrix elements in the considered representation of spin matrices, one finds from Eq. (14)

$$
n=\frac{\mu+m h}{U(0)+m^{2} J(0)} .
$$

The obtained formulae (15), (16) result in the following expression for the thermodynamic potential density:

$$
\omega=-\frac{1}{2} \frac{(\mu+m h)^{2}}{U(0)+m^{2} J(0)} .
$$

We are now in a position to study the stability of possible ground states (15). In the considered approximation, the thermodynamic potential of the normal state is zero (the order parameter $\Psi_{\alpha}$ vanishes). Therefore, for the stability of the ground state under consideration, the density of thermodynamic potential must be negative, $\omega<0$ and, consequently, according to (17), we can write the necessary condition of thermodynamic stability,

$$
U(0)+m^{2} J(0)>0 .
$$

Let us find now such spin projections $m$, which correspond to the minimum of potential (17). For simplicity, we study the case of $h=0$ (or sufficiently weak $h$ ). Then,

$$
\omega=-\frac{\mu^{2}}{2} \frac{1}{U(0)+m^{2} J(0)}<0 .
$$

As it can be easily seen that in contrast to usual Bogoliubov's theory, in which $U(0)>0$ (the necessary condition of stability), the negative values of $U(0)$ are also permissible. Therefore, we have the following three situations:

1) $U(0)>0, J(0)>0$. In this case the requirement (18) is automatically satisfied. The density of thermodynamic potential (17) has a minimum at $m=0$ in which $\omega=-\mu^{2} / 2 U(0)$. We call this case as antiferromagnetic ordering.

2) $U(0)>0, J(0)<0$ but such that the requirement (18) should be satisfied. The minimum of $\omega$ is reached for $m_{\min }= \pm\left(m_{\mathrm{c}}-1\right)$, where

$$
m_{\mathrm{c}}=\left[\left(-\frac{U(0)}{J(0)}\right)\right]^{1 / 2}, \quad m_{\mathrm{c}} \leq S+1
$$

(the square brackets denote an integer part). This case corresponds to ferromagnetic ordering. 
Spin-S Bose condensate in a magnetic field

3) $U(0)<0, J(0)>0$ but again, such that $U(0)+m^{2} J(0)>0$. Here the minimum of $\omega$ is given by the spin projections $m_{\min }= \pm\left(m_{\mathrm{c}}+1\right)$, where $m_{\mathrm{c}}$ is also defined by (19) but with $U(0)<0, J(0)>0$. This case also corresponds to ferromagnetic ordering.

\section{Low-lying collective modes}

In this section we obtain the excitation spectra of spin- $S$ BEC by employing the wellknown diagonalization procedure (Bogoliubov's $u-v$ transformations [10]) for the Hamiltonian quadratic in creation and annihilation operators. Note that the excitation spectra can also be found as a result of the linearization of the Gross-Pitaevskii equation [24, 25] for the condensate wave function (see e.g. [12]).

The part of the spin-exchange interaction Hamiltonian (9), which is quadratic in $\hat{a}_{\mathbf{p} \alpha},(\mathbf{p} \neq 0)$, has the form

$\hat{\mathcal{H}}_{\mathrm{e}}^{(2)}=J(0) \Psi^{*} \hat{\mathbf{S}} \Psi \sum_{\mathbf{p}} \hat{a}_{\mathbf{p}}^{\dagger} \hat{\mathbf{S}} \hat{a}_{\mathbf{p}}+\frac{1}{2} \sum_{\mathbf{p}} J(\mathbf{p})\left[\left(\hat{a}_{\mathbf{p}}^{\dagger} \hat{\mathbf{S}} \Psi\right)\left(\hat{a}_{-\mathbf{p}}^{\dagger} \hat{\mathbf{S}} \Psi\right)+\left(\hat{a}_{\mathbf{p}}^{\dagger} \hat{\mathbf{S}} \Psi\right)\left(\Psi^{*} \hat{\mathbf{S}} \hat{a}_{\mathbf{p}}\right)+\right.$ h.c. $]$,

where we have used the notations (12). Taking into account that $\hat{S}_{x}=\frac{1}{2}\left(\hat{S}_{+}+\hat{S}_{-}\right)$, $\hat{S}_{y}=-\frac{i}{2}\left(\hat{S}_{+}-\hat{S}_{-}\right)$and bearing in mind (10) for non-zero matrix elements of $\hat{S}_{ \pm}$as well as the explicit form of the condensate wave function $\Psi_{\alpha}^{(m)}=\sqrt{n} \delta_{\alpha m}$, one gets

$$
\begin{aligned}
& \left(\Psi^{*} \hat{\mathbf{S}} \Psi\right)\left(\hat{a}_{\mathbf{p}}^{\dagger} \hat{\mathbf{S}} \hat{a}_{\mathbf{p}}\right)=n m \sum_{\alpha} \alpha \hat{a}_{\mathbf{p} \alpha}^{\dagger} \hat{a}_{\mathbf{p} \alpha}, \\
& \left(\hat{a}_{\mathbf{p}}^{\dagger} \hat{\mathbf{S}} \Psi\right)\left(\hat{a}_{-\mathbf{p}}^{\dagger} \hat{\mathbf{S}} \Psi\right)=n m^{2} \hat{a}_{\mathbf{p} m}^{\dagger} \hat{a}_{-\mathbf{p} m}^{\dagger}+\frac{n}{2} S_{m} S_{-m}\left(\hat{a}_{\mathbf{p} m-1}^{\dagger} \hat{a}_{-\mathbf{p} m+1}^{\dagger}+\hat{a}_{\mathbf{p} m+1}^{\dagger} \hat{a}_{-\mathbf{p} m-1}^{\dagger}\right), \\
& \left(\hat{a}_{\mathbf{p}}^{\dagger} \hat{\mathbf{S}} \Psi\right)\left(\Psi^{*} \hat{\mathbf{S}} \hat{a}_{\mathbf{p}}\right)=n m^{2} \hat{a}_{\mathbf{p} m}^{\dagger} \hat{a}_{\mathbf{p} m}+\frac{n}{2}\left(S_{-m}^{2} \hat{a}_{\mathbf{p} m-1}^{\dagger} \hat{a}_{\mathbf{p} m-1}+S_{m}^{2} \hat{a}_{\mathbf{p} m+1}^{\dagger} \hat{a}_{\mathbf{p} m+1}\right) .
\end{aligned}
$$

where the following notation has been introduced:

$$
S_{m}=\sqrt{S(S+1)-m(m+1)} .
$$

Hence, $\hat{\mathcal{H}}_{\mathrm{e}}^{(2)}$ takes the form

$$
\begin{aligned}
\hat{\mathcal{H}}_{\mathrm{e}}^{(2)}=J(0) n m & \sum_{\mathbf{p}}\left[(m-1) \hat{a}_{\mathbf{p} m-1}^{\dagger} \hat{a}_{\mathbf{p} m-1}+m \hat{a}_{\mathbf{p} m}^{\dagger} \hat{a}_{\mathbf{p} m}+(m+1) \hat{a}_{\mathbf{p} m+1}^{\dagger} \hat{a}_{\mathbf{p} m+1}\right] \\
& +J(0) n m \sum_{\mathbf{p}, \alpha} \alpha \hat{a}_{\mathbf{p} \alpha}^{\dagger} \hat{a}_{\mathbf{p} \alpha}+\frac{n}{2} \sum_{\mathbf{p}} J(\mathbf{p}) m^{2}\left[\hat{a}_{\mathbf{p} m}^{\dagger} \hat{a}_{-\mathbf{p} m}^{\dagger}+2 \hat{a}_{\mathbf{p} m}^{\dagger} \hat{a}_{\mathbf{p} m}+\hat{a}_{-\mathbf{p} m} \hat{a}_{\mathbf{p} m}\right] \\
& +\frac{n}{2} \sum_{\mathbf{p}} J(\mathbf{p})\left[S_{m} S_{-m}\left(\hat{a}_{\mathbf{p} m-1}^{\dagger} \hat{a}_{-\mathbf{p} m+1}^{\dagger}+\hat{a}_{-\mathbf{p} m+1} \hat{a}_{\mathbf{p} m-1}\right)\right] \\
& +\frac{n}{2} \sum_{\mathbf{p}} J(\mathbf{p})\left[S_{-m}^{2} \hat{a}_{\mathbf{p} m-1}^{\dagger} \hat{a}_{\mathbf{p} m-1}+S_{m}^{2} \hat{a}_{\mathbf{p} m+1}^{\dagger} \hat{a}_{\mathbf{p} m+1}\right], \quad \alpha \neq m-1, m, m+1 .
\end{aligned}
$$

In this formula, the summation index $\alpha$ in the second term takes all values of spin projections except $m-1, m$, and $m+1$ (these three projections we have separated off and written them as the first term in $\left.\hat{\mathcal{H}}_{\mathrm{e}}^{(2)}\right)$. In a similar manner, keeping the terms only of second order in $\hat{a}_{\mathbf{p} \alpha}$, one finds according to (7), (8)

$\hat{\mathcal{H}}_{0}^{(2)}=\sum_{\mathbf{p}}\left[\left(\varepsilon_{p}-\mu\right)\left(\hat{a}_{\mathbf{p} m-1}^{\dagger} \hat{a}_{\mathbf{p} m-1}+\hat{a}_{\mathbf{p} m}^{\dagger} \hat{a}_{\mathbf{p} m}+\hat{a}_{\mathbf{p} m+1}^{\dagger} \hat{a}_{\mathbf{p} m+1}\right)\right]$ 
Spin-S Bose condensate in a magnetic field

$$
\begin{aligned}
& -h \sum_{\mathbf{p}}\left[(m-1) \hat{a}_{\mathbf{p} m-1}^{\dagger} \hat{a}_{\mathbf{p} m-1}+m \hat{a}_{\mathbf{p} m}^{\dagger} \hat{a}_{\mathbf{p} m}+(m+1) \hat{a}_{\mathbf{p} m+1}^{\dagger} \hat{a}_{\mathbf{p} m+1}\right] \\
& +\sum_{\mathbf{p}, \alpha}\left(\varepsilon_{p}-\mu-\alpha h\right) \hat{a}_{\mathbf{p} \alpha}^{\dagger} \hat{a}_{\mathbf{p} \alpha}, \quad \alpha \neq m-1, m, m+1,
\end{aligned}
$$

and

$$
\begin{aligned}
\hat{\mathcal{H}}_{\mathbf{p}}^{(2)}=U(0) n & \sum_{\mathbf{p}}\left[\hat{a}_{\mathbf{p} m-1}^{\dagger} \hat{a}_{\mathbf{p} m-1}+\hat{a}_{\mathbf{p} m}^{\dagger} \hat{a}_{\mathbf{p} m}+\hat{a}_{\mathbf{p} m+1}^{\dagger} \hat{a}_{\mathbf{p} m+1}\right]+U(0) n \sum_{\mathbf{p}, \alpha} \hat{a}_{\mathbf{p} \alpha}^{\dagger} \hat{a}_{\mathbf{p} \alpha} \\
& +\frac{n}{2} \sum_{\mathbf{p}} U(\mathbf{p})\left[\hat{a}_{\mathbf{p} m}^{\dagger} \hat{a}_{-\mathbf{p} m}^{\dagger}+2 \hat{a}_{\mathbf{p} m}^{\dagger} \hat{a}_{\mathbf{p} m}+\hat{a}_{-\mathbf{p} m} \hat{a}_{\mathbf{p} m}\right], \quad \alpha \neq m-1, m, m+1 .
\end{aligned}
$$

When obtaining (21) we have employed the fact that $\mathbf{h}$ is directed along $z$-axis, $\mathbf{h}=(0,0, h)$. Next, using Eq. (16) to eliminate the chemical potential $\mu$ in (21), we recast the total Hamiltonian $\hat{\mathcal{H}}^{(2)}=\hat{\mathcal{H}}_{0}^{(2)}+\hat{\mathcal{H}}_{\mathrm{p}}^{(2)}+\hat{\mathcal{H}}_{\mathrm{e}}^{(2)}$ that is quadratic in creation and annihilation operators in the following form:

$$
\hat{\mathcal{H}}^{(2)}=\hat{\mathcal{H}}_{\alpha}^{(2)}+\hat{\mathcal{H}}^{(2)}(m)+\hat{\mathcal{H}}^{2}(m-1, m+1),
$$

where

$$
\begin{gathered}
\hat{\mathcal{H}}_{\alpha}^{(2)}=\sum_{\mathbf{p}, \alpha}\left[\varepsilon_{p}-J(0) n m(m-\alpha)+h(m-\alpha)\right] \hat{a}_{\mathbf{p} \alpha}^{\dagger} \hat{a}_{\mathbf{p} \alpha}, \quad \alpha \neq m-1, m, m+1, \\
\hat{\mathcal{H}}^{(2)}(m)=\sum_{\mathbf{p}}\left[\varepsilon_{p}+g_{m}(\mathbf{p})\right] \hat{a}_{\mathbf{p} m}^{\dagger} \hat{a}_{\mathbf{p} m}+\frac{1}{2} \sum_{\mathbf{p}} g_{m}(\mathbf{p})\left[\hat{a}_{\mathbf{p} m}^{\dagger} \hat{a}_{-\mathbf{p} m}^{\dagger}+\hat{a}_{\mathbf{p} m} \hat{a}_{-\mathbf{p} m}\right], \\
\hat{\mathcal{H}}^{(2)}(m-1, m+1)=\sum_{\mathbf{p}}\left[\varepsilon_{p}-h+\beta_{m}(\mathbf{p})\right] \hat{a}_{\mathbf{p} m+1}^{\dagger} \hat{a}_{\mathbf{p} m+1} \\
\quad+\sum_{\mathbf{p}}\left[\varepsilon_{p}+h+\beta_{-m}(\mathbf{p})\right] \hat{a}_{\mathbf{p} m-1}^{\dagger} \hat{a}_{\mathbf{p} m-1} \\
\quad+\sum_{\mathbf{p}} \alpha_{m}(\mathbf{p})\left[\hat{a}_{\mathbf{p} m-1}^{\dagger} \hat{a}_{-\mathbf{p} m+1}^{\dagger}+\hat{a}_{\mathbf{p} m-1} \hat{a}_{-\mathbf{p} m+1}\right] .
\end{gathered}
$$

The introduced quantities $\alpha_{m}(\mathbf{p}), \beta_{m}(\mathbf{p})$, and $g_{m}(\mathbf{p})$ are given by

$$
\begin{aligned}
& \alpha_{m}(\mathbf{p})=\frac{n}{2} J(\mathbf{p}) S_{m} S_{-m}, \\
& \beta_{m}(\mathbf{p})=\frac{n}{2} J(\mathbf{p}) S_{m}^{2}+n J(0) m, \\
& g_{m}(\mathbf{p})=n\left(U(\mathbf{p})+m^{2} J(\mathbf{p})\right) .
\end{aligned}
$$

Now we are in a position to carry out the diagonalization procedure of the total Hamiltonian (23) quadratic in creation and annihilation operators. In this connection we note that the "Hamiltonians" (24)-(26) contain the creation and annihilation operators with not overlapping sets of indices $\alpha, m, m-1, m+1(\alpha \neq m-1, m, m+1)$. Therefore, we can perform their diagonalization independently. The evidence of this statement also follows from the fact that (23) can be considered as the Hamiltonian of the system consisting of four kinds $(m, m \pm 1, \alpha)$ of noninteracting particles.

The "Hamiltonian" $\hat{\mathcal{H}}_{\alpha}^{(2)}$ has already a diagonal form with the following spectrum:

$$
\omega_{m, \alpha}(\mathbf{p})=\varepsilon_{p}-J(0) n m(m-\alpha)+h(m-\alpha) .
$$


Spin-S Bose condensate in a magnetic field

To carry out the diagonalization of $\hat{\mathcal{H}}^{(2)}(m-1, m+1)$, we introduce the creation and annihilation operators $\hat{b}_{\mathbf{p} m+\sigma}(\sigma= \pm 1)$,

$$
\begin{aligned}
& \hat{a}_{\mathbf{p} m+\sigma}=u_{m, \sigma}(\mathbf{p}) \hat{b}_{\mathbf{p} m+\sigma}+v_{m, \sigma}(\mathbf{p}) \hat{b}_{-\mathbf{p} m-\sigma}^{\dagger}, \\
& \hat{a}_{\mathbf{p} m+\sigma}^{\dagger}=u_{m, \sigma}^{*}(\mathbf{p}) \hat{b}_{\mathbf{p} m+\sigma}^{\dagger}+v_{m, \sigma}^{*}(\mathbf{p}) \hat{b}_{-\mathbf{p} m-\sigma},
\end{aligned}
$$

in terms of which it has the diagonal form,

$$
\hat{\mathcal{H}}^{(2)}(m-1, m+1)=\sum_{\mathbf{p}, \sigma} \omega_{m, \sigma}(\mathbf{p}) \hat{b}_{\mathbf{p} m+\sigma}^{\dagger} \hat{b}_{\mathbf{p} m+\sigma}+E_{0}
$$

where $\omega_{m, \sigma}(\mathbf{p})$ and $E_{0}$ are the excitation spectrum and the ground state energy respectively. In order that the introduced operators $\hat{b}_{\mathbf{p} m+\sigma}^{\dagger}, \hat{b}_{\mathbf{p} m+\sigma}$ meet the canonical commutation relations, the functions $u_{m, \sigma}(\mathbf{p}), v_{m, \sigma}(\mathbf{p})$ must obey the relationships

$$
\begin{aligned}
& \left|u_{m, \sigma}(\mathbf{p})\right|^{2}-\left|v_{m, \sigma}(\mathbf{p})\right|^{2}=1, \\
& u_{m, \sigma}(\mathbf{p}) v_{m,-\sigma}(-\mathbf{p})-v_{m, \sigma}(\mathbf{p}) u_{m,-\sigma}(-\mathbf{p})=0 .
\end{aligned}
$$

Next, noting that

$$
\left[\hat{\mathcal{H}}^{(2)}(m-1, m+1), \hat{a}_{\mathbf{p} m+\sigma}\right]=-\alpha_{m}(\mathbf{p}) \hat{a}_{-\mathbf{p} m-\sigma}^{\dagger}-\gamma_{m, \sigma} \hat{a}_{\mathbf{p} m+\sigma}
$$

and expressing the right-hand side of this formula through $\hat{b}_{\mathbf{p} m+\sigma}$, one gets

$$
\begin{aligned}
{\left[\hat{\mathcal{H}}^{(2)}(m-1, m+1), \hat{a}_{\mathbf{p} m+\sigma}\right]=} & -\left(\alpha_{m}(\mathbf{p}) v_{m,-\sigma}^{*}(-\mathbf{p})+\gamma_{m, \sigma} u_{m, \sigma}(\mathbf{p})\right) \hat{b}_{\mathbf{p} m+\sigma} \\
& -\left(\alpha_{m}(\mathbf{p}) u_{m,-\sigma}^{*}(-\mathbf{p})+\gamma_{m, \sigma} v_{m, \sigma}(\mathbf{p})\right) \hat{b}_{-\mathbf{p} m-\sigma}^{\dagger},
\end{aligned}
$$

where $\gamma_{m, \sigma}$ is defined by

$$
\gamma_{m, \sigma}=\varepsilon_{p}+\beta_{m \sigma}(\mathbf{p})-\sigma h,
$$

moreover $\beta_{m \sigma}(\mathbf{p})$ depends on the product $m \sigma$. On the other hand, the straightforward use of (32), (31) results in

$$
\left[\hat{\mathcal{H}}^{(2)}(m-1, m+1), \hat{a}_{\mathbf{p} m+\sigma}\right]=v_{m, \sigma}(\mathbf{p}) \omega_{m,-\sigma}(-\mathbf{p}) \hat{b}_{-\mathbf{p} m-\sigma}^{\dagger}-u_{m, \sigma}(\mathbf{p}) \omega_{m, \sigma}(\mathbf{p}) \hat{b}_{\mathbf{p} m+\sigma} .
$$

The comparison of (34) with (36) gives the coupled equations for $u_{m, \sigma}(\mathbf{p})$ and $v_{m,-\sigma}^{*}(-\mathbf{p})$,

$$
\begin{gathered}
\left(\gamma_{m, \sigma}-\omega_{m, \sigma}(\mathbf{p})\right) u_{m, \sigma}(\mathbf{p})+\alpha_{m}(\mathbf{p}) v_{m,-\sigma}^{*}(-\mathbf{p})=0, \\
\alpha_{m}(\mathbf{p}) u_{m, \sigma}(\mathbf{p})+\left(\gamma_{m,-\sigma}+\omega_{m, \sigma}(\mathbf{p})\right) v_{m,-\sigma}^{*}(-\mathbf{p})=0 .
\end{gathered}
$$

The condition for the existence of non-trivial solutions to the coupled Eqs. (37) along with the definitions (27)-(29), (35) result in the following expression for the excitation spectrum:

$$
\begin{aligned}
& \omega_{m, \sigma}(\mathbf{p})=n m \sigma\left(J(0)-\frac{1}{2} J(\mathbf{p})\right) \\
& \pm\left[\varepsilon_{p}^{2}+\varepsilon_{p} n J(\mathbf{p})\left(S(S+1)-m^{2}\right)+\left(\frac{n J(\mathbf{p}) m}{2}\right)^{2}\right]^{1 / 2}-\sigma h
\end{aligned}
$$

At small $\mathbf{p}$ and $J(0)<0$ the obtained spectrum is real (the state is stable) if the spin projections $m$ meet the inequality $m^{2}>m_{c}^{2}$, where

$$
m_{c}=\left[\left(\frac{S(S+1)}{1-n J(0) / 4 \varepsilon_{p}}\right)\right]^{1 / 2}, \quad m_{c} \leq S
$$


and the square brackets, as in (19), are introduced to denote an integer part.

The functions $u_{m, \sigma}(\mathbf{p}), v_{m, \sigma}(\mathbf{p})$ are found from (33), (37) and have the form

$$
\begin{aligned}
& u_{m, \sigma}(\mathbf{p})=\frac{\alpha_{m}(\mathbf{p})}{\sqrt{\alpha_{m}^{2}(\mathbf{p})-\left(\omega_{m, \sigma}(\mathbf{p})-\gamma_{m, \sigma}\right)^{2}}}, \\
& v_{m, \sigma}(\mathbf{p})=\frac{\omega_{m, \sigma}(\mathbf{p})-\gamma_{m, \sigma}}{\sqrt{\alpha_{m}^{2}(\mathbf{p})-\left(\omega_{m, \sigma}(\mathbf{p})-\gamma_{m, \sigma}\right)^{2}}} .
\end{aligned}
$$

In fact, the functions $u_{m, \sigma}(\mathbf{p}), v_{m, \sigma}(\mathbf{p})$ do not depend on $\sigma$ because, as it can be easily shown, the quantity $\omega_{m, \sigma}(\mathbf{p})-\gamma_{m, \sigma}$ is independent of $\sigma$. The sign plus before the square root in (38) corresponds (for $\sigma=1$ ) to the wave, which propagates in one direction, whereas the sign minus corresponds (for $\sigma=-1$ ) to the wave propagating in opposite direction. Notice that the obtained spectrum, as well as (30), contains only the spin-exchange interaction amplitude and does not depend on the potential interaction amplitude.

When $m=0$ (the antiferromagnetic ordering), the excitation spectrum (38) takes the form

$$
\omega(\mathbf{p})=\sqrt{\varepsilon_{p}^{2}+\varepsilon_{p} n J(\mathbf{p}) S(S+1)} \pm h .
$$

In this case, for $h=0$ and $p \rightarrow 0$ we have

$$
\omega(p)=c p, \quad c=\sqrt{\frac{n}{2 M} J(0) S(S+1)} .
$$

In ferromagnetic case (when $m=S$ ) the excitation spectrum is of the form

$$
\omega(\mathbf{p})=\varepsilon_{p}+\frac{n J(\mathbf{p}) S}{2}(1-\sigma)+n J(0) S \sigma-\sigma h .
$$

The similar mathematical manipulations with $\hat{\mathcal{H}}^{(2)}(m)$ lead to another mode of excitation spectrum, which depends both on potential and spin-exchange interaction amplitudes,

$$
\omega_{m}(\mathbf{p})=\sqrt{\varepsilon_{p}^{2}+2 \varepsilon_{p} n\left(U(\mathbf{p})+m^{2} J(\mathbf{p})\right)} .
$$

The stability region for this spectrum at small $\mathbf{p}$ is given by (19).

The functions $u_{m}(\mathbf{p})$ and $v_{m}(\mathbf{p})$ can be found immediately,

$$
u_{m}(\mathbf{p})=\frac{\varepsilon_{p}+\omega_{m}(\mathbf{p})}{2 \sqrt{\varepsilon_{p} \omega_{m}(\mathbf{p})}}, \quad v_{m}(\mathbf{p})=\frac{\omega_{m}(\mathbf{p})-\varepsilon_{p}}{2 \sqrt{\varepsilon_{p} \omega_{m}(\mathbf{p})}} .
$$

When $J(\mathbf{p})=0$ the excitation spectrum (39) coincides with the spectrum found by Bogoliubov [10]. At small $\mathbf{p}$, the spectrum has the following phonon behavior:

$$
\omega_{m}(\mathbf{p})=c p, \quad c=\sqrt{\frac{n}{M}\left(U(0)+m^{2} J(0)\right)} .
$$

In this formula, as well as in (39), we have chosen the arithmetic value of the square root.

In conclusion, we have studied BEC of atoms with arbitrary spin in a magnetic field on the basis of the model for weakly interacting Bose gas. We have derived the equation 
which describes the ground state of spin- $S$ BEC at temperature $T \rightarrow 0$ and found its particular solution. This solution corresponds to the formation of BEC of spin- $S$ atoms with a definite spin projection $m$ onto direction of a magnetic field that is also true for an ideal Bose gas [11]. The explicit expression for thermodynamic potential being a function of chemical potential and spin projection has been obtained. It generalizes the thermodynamic potential for weakly interacting Bose gas to the case when both potential and spin-exchange interactions act between bosons. The thermodynamic stability of the state under consideration has been studied and the spin projections, which give a minimum of thermodynamic potential, have been found. These projections are given by the integer part of the ratio of potential to spin-exchange interaction amplitude. The expressions for low-lying collective modes related to the solution (15) have been obtained. Notice that Eq. (14) for order parameter has also other solutions different from (15). Our present research deals with seeking such solutions.

\section{Acknowledgments}

A. Peletminskii would like to thank the Abdus Salam ICTP (Trieste, Italy) for the support, warm hospitality, and stimulating research environment during the visit to the Centre within the framework of Associateship Scheme in summer 2005. He is also grateful to Professor S. Shenoy for his attention to the work.

\section{References}

[1] M.H. Anderson, J.R. Ensher, M.R. Matthews, C.E. Wieman, E.A. Cornell, Science 269 (1995) 198.

[2] K.B. Davis, M.-O. Mewes, M.R. Andrews, N.J. van Druten, D.S. Durfee, D.M. Kurn, W. Ketterle, Phys. Rev. Lett. 75 (1995) 3969.

[3] C.C. Bradley, C.A. Sackett, J.J. Tollett, R.G. Hulet, Phys. Rev. Lett. 75 (1995) 1687.

[4] C.J. Pethick, H. Smith, Bose-Einstein Condensation in Dilute Gases, Cambridge: Cambridge University Press, 2002.

[5] L. Pitaevskii, S. Stringari, Bose-Einstein Condensation, New York: Oxford University Press, 2003.

[6] D.G. Fried, T.C. Killian, L. Willmann, D. Landhuis, S.C. Moss, D. Kleppner, T.J. Greytak, Phys. Rev. Lett. 81 (1998) 3811.

[7] F. Pereira dos Santos, J. Lonard, J. Wang, C.J. Barrelet, F. Perales, E. Rasel, C.S. Unikrishnan, M. Leduc, C. Cohen-Tannoudji, Phys. Rev. Lett. 86 (2001) 3459.

[8] G. Modugno, G. Ferrari, G. Roati, R.J. Brecha, A. Simoni, M. Inguscio, Science 294 (2001) 1320.

[9] S. Chu, Rev. Mod. Phys. 70 (1998) 685; C.N. Cohen-Tannoudji, Rev. Mod. Phys. 70 (1998) 719; W.D. Phillips, Rev. Mod. Phys. 70 (1998) 721.

[10] N.N. Bogoliubov, J. Phys. (USSR) 11 (1947) 23.

[11] A.I. Akhiezer, S.V. Peletminskii, Yu.V. Slyusarenko, JETP 86 (1998) 501.

[12] T. Ohmi, K. Machida, J. Phys. Soc. Japan 67 (1998) 1822.

[13] T.-L. Ho, Phys. Rev. Lett. 81 (1998) 742.

[14] M. Ueda, Phys. Rev. A 63 (2000) 013601.

[15] C.K. Law, H. Pu, N.P. Bigelow, Phys. Rev. Lett. 81 (1998) 5257.

[16] M. Koashi, M. Ueda, Phys. Rev. Lett. 84 (2000) 1066.

[17] T.-L. Ho, S.-K. Yip, Phys. Rev. Lett. 84 (2000) 4031.

[18] M. Ueda, M. Koashi, Phys. Rev. A 65 (2002) 063602. 
[19] J.-P. Martikainen, K.-A. Suominen, J. Phys. B: At. Mol. Opt. Phys. 34 (2001) 4091.

[20] D.M. Stamper-Kurn, M.R. Andrews, A.P. Chikkatur, S. Inouye, H.-J. Miesner, J. Stenger, W. Ketterle, Phys. Rev. Lett. 80 (1998) 2027.

[21] N.N. Bogoliubov, Quasiaverages in Problems of Statistical Mechanics, Preprint 145 (1963) OIYaI Dubna.

[22] N.N. Bogoliubov, Lectures on Quantum Statistics, vol. 2, Quasi-averages, New York: Gordon and Breach, 1970.

[23] A.I. Akhiezer, S.V. Peletminskii, Methods of Statistical Physics, Oxford: Pergamon Press, 1981.

[24] E.P. Gross, Nuovo Cimento, 20 (1961) 451; E.P. Gross, J. Math. Phys. 4 (1963) 195.

[25] L.P. Pitaevskii, Sov. Phys. JETP, 13 (1961) 451. 